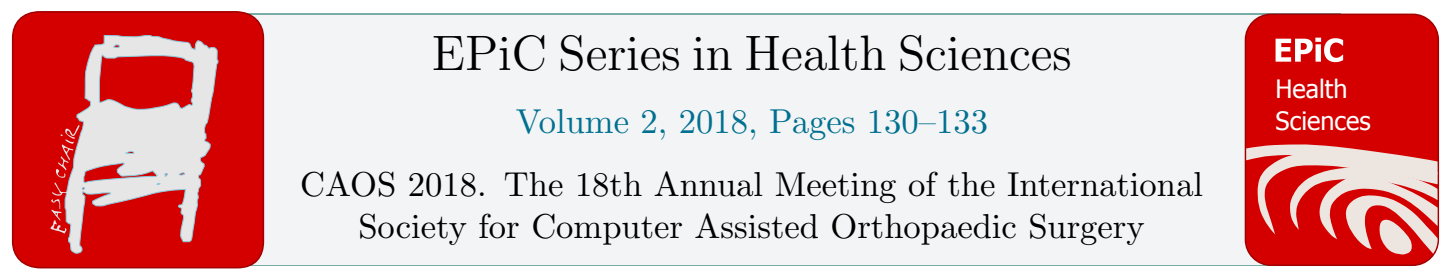

\title{
The Study of Artificial Intelligence-Assisted Diagnosis of Low Back Pain
}

\author{
Chunde Li MD ${ }^{1 *}$, Longtao Qi MD 2 , Yangyang Xu MD ${ }^{3}$ \\ 1,2 Department of Orthopedics of Peking University 1st Hospital, Beijing, 100034, China, \\ q1t6219@126.com \\ ${ }^{3}$ Department of Orthopedics of Peking University 1st Hospital, Beijing, 100034, China.
}

\begin{abstract}
Low back pain is one of the most common reasons for outpatient service. It is associated with high costs, and can cause loss of labor and the indirect economic. In Europe and the United States, lower back pain has the corresponding guideline for diagnosis and treatment. But, the hierarchical medical system and process of China is not established at present, and there is lack of guidelines. So, a large number of patients with low back pain have outpatient service in the superior hospital, and it caused a conflict between the increasing demand for outpatient visits and limited medical resources and wasted a lot of public health resources. So, we want to apply the artificial intelligence on the diagnosis of low back pain to help doctor diagnosis and reduce the burden of clinic work.
\end{abstract}

\section{Introduction}

Low back pain is one of the most common reasons for outpatient service. The articles reported that approximately one quarter of American adults had low back pain lasting at least 1 day in the past 3 months ${ }^{[1]}$. Low back pain is associated with high costs, and the total costs attributable to low back pain in the United States were estimated at $\$ 100$ billion $^{[2]}$, most of which were indirect costs of lost wages and productivity ${ }^{[3]}$. In China, although there is no accurate statistical data of the loss of labor and the indirect economic caused by low back pain, it must be very great. At present, the hierarchical medical system and process of China is not established, and a large number of patients with low back pain have outpatient service in the superior hospital. According to the date of National Health and Family 
Planning Commission, the superior hospitals are only $7.66 \%$ of all hospitals, but the percent of outpatient service in the superior hospital is $55.9 \%{ }^{[4]}$. It caused a conflict between the increasing demand for outpatient visits and limited medical resources and wasted a lot of public health resources.

Although the computer-assisted orthopedic surgery was(CAOS) implemented since the 90 's, the computer technology has broadly applied pre-, intra- and/or post-operatively to improve the outcome of orthopedic surgical procedures. The research of artificial intelligence in surgery had made a great progress, and the artificial intelligence applied on the diagnosis had been a hot topic. Such as image diagnosis and pathological diagnosis of disease. Recently, the Waston system of IBM is the most mature intelligent diagnosis system [5]. It can read 3469 monographs, 248000 papers, 69 kinds of treatments and 106000 clinic medical reports in 17 seconds, and can provide auxiliary diagnosis and treatment services to help the doctor and patient to choose the best treatment in hospitals of the United States. So we apply the artificial intelligence on the diagnosis of low back pain to help doctor diagnosis and reduce the burden of clinic work. The artificial intelligence-assisted diagnosis system of low back pain which we had built was named the AiKNOWN system.

\section{Methods}

\section{To bulid and consummate the AiKNOWN system}

Before building the AiKNOWN system, we first builded a medical knowledge graph of low back pain. The knowledge graph was based on European and American guidelines of the low back pain and the medical record data. Firstly, we used the natural language technology (NLP) to identify the words in the medical records and annotate keywords. The words identified from the medical records were called the entity data. After obtaining the entity data, we used the dependence analysis and semantic parsing syntactic structure to help identify the relationship between the two entities. Then, we used the unified terminology to fuse the different knowledge text data, and used the processed data to build knowledge graph. Based on the knowledge graph, we combined the rules engine based on symbol reasoning and the statistical engine based on machine learning to make knowledge reasoning of medical record data. The rules engine based on the default logic can use the rules and the existing knowledge graph to find the new relationship between entity data, and detect the logical conflict. Statistical engine can use the new relationship between entities to find valid data hiding in medical record data. After all above have done, the AiKNOWN system is built.

After the system was built, we use 500 medical records for AiKNOWN system to learn. Firstly, the AiKNOWN system uses its own knowledge map to analyze patient data and generate a diagnostic model frame. Then, the system gives a diagnosis and verify the diagnosis of AiKNOWN system given to the final diagnosis of medical record. Thirdly, the AiKNOWN system studies the results of the validation through feedback and learning mechanism. Lastly, the system adjusts the diagnostic model frame to make a new model and repeat the above cycle until obtaining a more accurate diagnostic model. 


\section{Assistant of the orthopedic doctor and the diagnostic consistency assessment}

When the patients wait for outpatient service, they can fill in their history under the help of AiKNOWN system. Firstly, we will obtain the history and symptom of the patients with low back pain with the AiKNOWN system, include the main symptom, medical history, personal history, and other disease history. Secondly, the system gives a diagnosis. and three senior doctors also give the diagnosis independently. Thirdly, we use the diagnostic results of the doctor as the gold standard, and the diagnostic consistency of AiKNOWN system was evaluated. The definition of the doctor's diagnosis: if three doctors have the same diagnosis, the result is standard; If the results of the three doctors' diagnosis were different, the conclusion was reached after discussion, and the result was the standard.

The diagnostic consistency is evaluated by coincidence rate, which means the consistency of diagnosis between the AiKNOWN system and the doctors.

\section{Results And Discussion}

When we built the AiKNOWN system, the common diseases which caused low back pain were incorporated into the knowledge graph, such as cancer, compression fracture, spinal infection, ankylosing spondylitis, spinal stenosis, symptomatic herniated disc, the cauda equina syndrome, and nonspecific low back pain. After the study of 500 medical records of low back pain, the coincidence rate of diagnosis for AiKNOWN system is $74 \%$ compared with the final diagnosis of medical records.

At the outpatient department, 106 patients with low back pain were incorporated into to evaluate the we evaluated the consistency of diagnosis for low back pain between the AiKNOWN system and the doctors. The coincidence rate of diagnosis for AiKNOWN system is $74.6 \%$ compared with the final diagnosis of doctors.

We wanted to evaluate the total diagnostic consistency of AiKNOWN system, such as coincidence rate, Kappa coefficient and 95\% confidence interval, and the diagnostic consistency and accuracy of AiKNOWN system for each disease caused low back pain, such as sensitivity, specificity, positive predictive value, negative predictive value, coincidence rate and Kappa coefficient. But the number of patients with low back pain was not enough, we couldn't finish the statistics analysis. In the future, we will continue consummate the AiKNOWN system by learning 5000 medical records of low back pain, and we will incorporate more patients with low back pain to evaluate the the diagnostic consistency and accuracy of AiKNOWN system. The final coincidence rate of diagnosis for AiKNOWN system we expect is $85 \%$.

\section{Conclusion and Future Work}

In the Preliminary study, the artificial intelligence shows a certain value in the diagnosis of low back pain. But it still need more medical records for AiKNOWN system to learn and consummate. This is the key work in our future work. 


\section{References}

Deyo RA, Mirza SK, Martin BI. Back pain prevalence and visit rates: estimates from U.S. national surveys, 2002. Spine (Phila Pa 1976). 2006;31:2724-7.

Katz JN. Lumbar disc disorders and low-back pain: socioeconomic factors and consequences. J Bone Joint Surg Am. 2006;88 Suppl 2:21-4.

Luo X, Pietrobon R, Sun SX, Liu GG, Hey L. Estimates and patterns of direct health care expenditures among individuals with back pain in the United States. Spine 2004; 29(1):79-86.

National health and family planning commission information center, www.nhfpc.gov.cn

Artificial Intelligence and Medical Research: Time to Aim Higher? Rejuvenation Res. 2016 Apr;19(2):105-6 